\title{
Non-normal cone metric and cone $b$-metric spaces and fixed point results
}

\author{
Z. Kadelburg, Lj. Paunović, S. Radenović, G. Soleimani Rad
}

\begin{abstract}
We show that most fixed point results obtained so far in cone metric spaces over solid non-normal cones can be easily reduced to the case of solid normal cones and, hence, their proofs can be made much simpler. Also, cone tvs-valued spaces over solid cones are not an essential generalization of cone metric spaces. These results are consequences of the simple fact that each solid cone in a topological vector space is in fact normal under a suitably defined norm. The proof follows by using the technique of Minkowski functional. As an application of these results, we prove an extension of the classical Nemytzki-Edelstein fixed point result to (tvs)-(b)-cone metric spaces over solid cones.
\end{abstract}

Keywords: Topological vector space; ordered normed space; cone metric space; $b$-metric space; tvs-cone $b$-metric space; Minkowski functional.

\section{Introduction}

The connection between cones and order relations in vector spaces is very well known. In particular, the usage of ordered normed spaces in Functional Analysis date back to 1940's (see $[24,25,30,31])$. It seems that Kurepa ([26]) was the first to use ordered normed spaces as the codomain of a metric (see [28]). Later on, such "metric" spaces appeared occasionally under various names: $K$-metric spaces, abstract metric space, generalized metric spaces (see, e.g., [32]).

The spaces of this type were re-introduced in 2007 by Huang and Zhang [16] under the name of cone metric spaces. Among other things, they used the relation $\ll$ (mentioned already in [24]) which could be defined under the supposition that the underlying cone had a nonempty interior (such cones are usually called solid). Later, these definitions were

Manuscript received April 30, 2016; accepted September 2, 2016.

Z. Kadelburg is with the Faculty of Mathematics, University of Belgrade, Beograd, Serbia; Lj. Paunović is with the Teacher Education School in Prizren-Leposavić, Leposavić, Serbia; S. Radenović is with the State University of Novi Pazar, Novi Pazar, Serbia, G. Soleimani Rad is with the Young Researchers and Elite club, Central Tehran Branch, Islamic Azad University, Tehran, Iran. 
extended for topological vector space-valued cone metric space (or tvs-cone metric space) in $[4,10,21]$. Afterwards, several authors obtained a lot of fixed point results in (tvs)-cone metric spaces.

Several authors showed (by various methods) that each cone metric space over a solid cone is metrizable (see, e.g., [2, 5, 10, 15, 20, 23]). However, this does not mean that all fixed point results can be reduced in this way to their standard metric counterparts. For example, it is still not known whether scalar and vector versions of the celebrated Caristi's fixed point result are equivalent (see [22]).

In the paper [16], the underlying cones were supposed to be normal (see the definition in next section). It was shown in [29] that such assumption is sometimes not necessary. Hence, further, a lot of authors obtained (common) fixed point results for non-normal solid cones (see a survey of these results until 2011 in [19]; a lot of papers appeared afterwards, too). However, the respective proofs were usually rather long and not direct extensions of the known proofs from the standard metric case.

In this paper, we show that most fixed point results obtained so far in cone metric spaces over solid non-normal cones can be easily reduced to the case of solid normal cones and, hence, their proofs can be made much simpler. Also, cone tvs-valued spaces over solid cones are not an essential generalization of cone metric spaces. These results are consequences of the simple fact that each solid cone in a tvs is in fact normal under a suitably defined norm (not always equivalent with the original one). The proof follows by using the technique of Minkowski functional, already used in [20]. As an application of these results, we prove an extension of the classical Nemytzki-Edelstein fixed point result $[13,27]$ to tvs-cone metric spaces over solid cones.

Continuing in this direction, we consider tvs-cone $b$-metric spaces as a generalization of $b$-metric spaces and prove that some of fixed point theorems in cone $b$-metric spaces can be obtained in an easier way. Our method is even easier than that of Du and Karapinar [11] and can be considered as a continuation of the papers $[5,10,20]$.

\section{Preliminaries}

Throughout the paper, $(E, t)$ will be a real Hausdorff topological vector space (abbr. $t v s$ ) with the zero vector denoted as $\theta$. A cone in $E$ is a proper nonempty and closed subset $C$ which satisfies: $1^{\circ} C+C \subset C, 2^{\circ} \lambda C \subset C$ for $\lambda \geq 0$, and $2^{\circ} C \cap(-C)=\{\theta\}$. If the cone $C$ has a nonempty interior int $C$ then it is called solid.

Example 1 Some examples of solid cones are $\left\{x=\left(x_{i}\right)_{i=1}^{n} \in \mathbb{R}^{n}: x_{i} \geq 0, i=1, \ldots, n\right\}$ in $\mathbb{R}^{n}$ and $\{x \in C[a, b]: x(t) \geq 0, a \leq t \leq b\}$ in $C[a, b]$.

However, if one defines a cone in a similar manner in some other spaces, e.g., in $c_{0}, l^{p}$ $(p>0), L^{p}(p>0)$, it appears to have empty interior [8, 31$]$. 
Each cone $C$ defines a partial order $\preceq$ on the vector space $E$ by $x \preceq y \Leftrightarrow y-x \in C$. We will write $x \prec y$ when $x \preceq y$ and $x \neq y$. If the cone $C$ is solid, $x \ll y$ denotes that $y-x \in \operatorname{int} C$. The triple $(E, t, C)$ is an ordered topological vector space.

For a pair of elements $x, y \in E$ such that $x \preceq y$, put $[x, y]=\{z \in E: x \preceq z \preceq y\}$. The sets of the form $[x, y]$ are named order-intervals. A subset $A$ of $E$ is called order-convex if $[x, y] \subset A$ whenever $x, y \in A$ and $x \preceq y$. Ordered topological vector space is order-convex if it has a base of neighborhoods of $\theta$ consisting of order-convex subsets. In this case, the cone $C$ is called normal. When $E$ is a normed space, i.e., the topology $t$ is induced by a norm $\|\cdot\|$, the last condition is fulfilled if and only if its unit ball is order-convex. This can be equivalently expressed as: there is a number $m$ such that $x, y \in E$ and $\theta \preceq x \preceq y$ imply that $\|x\| \leq m\|y\|$. The minimal constant $m$ satisfying the previous condition is called the normal constant of $C$. Obviously, the normal constant is always greater or equal to 1 . In the case when $m=1$, i.e., when $\theta \preceq x \preceq y$ implies that $\|x\| \leq\|y\|$, the cone $C$ is called monotone.

Lemma 1 ([24], for the proof see [9, 31]) The following conditions are equivalent for a cone $C$ in the normed space $(E,\|\cdot\|)$ :

(1) C is normal;

(2) there exists a norm $\|\cdot\|_{1}$ on E, equivalent to the given norm $\|\cdot\|$, such that the cone C is monotone w.r.t. $\|\cdot\|_{1}$.

The following example is classical.

Example 2 Let $E=C_{\mathbb{R}}^{1}[0,1]$, with $\|x\|=\|x\|_{\infty}+\left\|x^{\prime}\right\|_{\infty}, C=\{x \in E: x(t) \geq 0, t \in[0,1]\}$. This cone is solid but non-normal. Consider, for example, $x_{n}(t)=\frac{t^{n}}{n}$ and $y_{n}(t)=\frac{1}{n}$. Then $\theta \preceq x_{n} \preceq y_{n}$, and $\lim _{n \rightarrow \infty} y_{n}=\theta$, but $\left\|x_{n}\right\|=\max _{t \in[0,1]}\left|\frac{t^{n}}{n}\right|+\max _{t \in[0,1]}\left|t^{n-1}\right|=\frac{1}{n}+1>1$; hence $\left\{x_{n}\right\}$ does not converge to zero. It follows that $C$ is a non-normal cone.

Definition $1[4,10,16,21]$ Let $X$ be a nonempty set and $(E, t, C)$ be an ordered tvs. Suppose that a function $p: X \times X \rightarrow E$ satisfies the following conditions:

(p1) $\theta \preceq p(x, y)$ for all $x, y \in X$ and $p(x, y)=\theta$ if and only if $x=y$;

(p2) $p(x, y)=p(y, x)$ for all $x, y \in X$;

(p3) $p(x, z) \preceq p(x, y)+p(y, z)$ for all $x, y, z \in X$.

Then $p$ is called a tvs-cone metric and $(X, p)$ is called a tvs-cone metric space. 


\section{Tvs-cone metric spaces}

Let $V$ be an absolutely convex and absorbing subset of a vector space $E$. Recall that its Minkowski functional is defined by $q_{V}(x)=\inf \{\lambda>0: x \in \lambda V\}$ for $x \in E$. It is a seminorm on $E$ (see, e.g. [30, II.1.5]) and $V \subset W$ implies that $q_{W}(x) \leq q_{V}(x)$ for $x \in E$.

Now, let $(E, t, C)$ be an ordered tvs with $C$ being solid, and let $e \in \operatorname{int} C$. Then $[-e, e]=$ $(C-e) \cap(e-C)=\{z \in E:-e \preceq z \preceq e\}$ is an absolutely convex neighborhood of $\theta$ (for the proof see, e.g., [31, Proposition 2.2]). Its Minkowski functional $q_{[-e, e]}$ will be denoted by $q_{e}$. Note that $\operatorname{int}[-e, e]=(\operatorname{int} C-e) \cap(e-\operatorname{int} C)$, and $q_{e}$ is an increasing function on $C$.

Lemma 2 The functional $q_{e}$ is a monotone norm on $(E, C)$, i.e., $C$ is a cone which is solid and normal w.r.t. $q_{e}$.

Proof In order to prove that $q_{e}$ is a norm on $E$, it suffices to show that $q_{e}(x)=0$ implies that $x=\theta$. Suppose that $q_{e}(x)=0$ for some $x \in E$. There is a sequence $\left\{\lambda_{n}\right\}$ of positive scalars such that $\lambda_{n} \rightarrow 0$ as $n \rightarrow \infty$ and $x \in \lambda_{n}[-e, e]$, i.e., $\lambda_{n} e-x \succeq \theta$ and $\lambda_{n} e+x \succeq \theta$. Passing to the limit as $n \rightarrow \infty$ and using that $C$ is a cone in the given $t v s(E, t)$, we get that $x=\theta$.

The obtained norm $q_{e}$ is obviously monotone, i.e., $\theta \preceq x \preceq y$ implies that $q_{e}(x) \leq q_{e}(y)$.

Solidness and closedness of $C$ w.r.t. $q_{e}$ are proved, e.g., in [9, Proposition 19.9] and [1, Theorem 2.55].

Remark 1 In the case when the given space $E$ is normed, we obtain in this way two norms on it: the original norm and $q_{e}$. It is important to notice that if $C$ is a normal cone w.r.t. the original norm, then these two norms are equivalent [9]. However, if this is not the case, then the two norms cannot be equivalent, because of Lemma 1 and Example 2.

Note also that if $e_{1}, e_{2}$ are two points from int $C$, then the norms $q_{e_{1}}$ and $q_{e_{2}}$ are always equivalent, i.e., they define the same topology. This topology is order-convex, i.e., it has a base of $\theta$-neighborhoods consisting of order-convex subsets. This follows from the fact that if the cone $C$ is normal (with the normal constant 1 ) and $e_{1}, e_{2} \in \operatorname{int} C$, then each of the order-intervals $\left[-e_{1}, e_{1}\right],\left[-e_{2}, e_{2}\right]$ absorbs the other.

Combining now Lemma 2 with Theorems 3.1 and 3.2 from [20], we deduce that:

(i) Each tvs-cone metric space with the underlying cone $C$ that is solid in an ordered tvs $(E, t, C)$ is in fact a cone metric space over (the same) cone $C$ which is normal in an appropriate ordered normed space $(E,\|\cdot\|, C)$.

(ii) Each cone metric space over a solid cone is also a cone metric space over a solid and normal cone (in an appropriate norm). 
We note that it is not clear whether these conclusions can be obtained using scalarization functions (which are not norms) that were utilized in several articles following [10].

We will apply now the obtained results to show how some cone metric versions of well known fixed point results can be obtained in an easy way. We first prove the following

Lemma 3 If $(E, t, C)$ is an ordered tvs with a solid cone $C$, and $e \in$ int $C$ is arbitrary, then

$$
\theta \preceq x \ll y \text { implies that } q_{e}(x)<q_{e}(y) .
$$

Proof Suppose that $x, y \in E$ and $\theta \preceq x \ll y$. Then, considering the sequence $\left\{\frac{1}{n} y\right\}$ (obviously converging to $\theta$ ) and $y-x \in \operatorname{int} C$, we get that

$$
\frac{1}{n} y \ll y-x, \quad \text { i.e., } \quad x \ll\left(1-\frac{1}{n}\right) y,
$$

for $n$ sufficiently large. It follows that

$$
q_{e}(x) \leq q_{e}\left(\left(1-\frac{1}{n}\right) y\right)=\left(1-\frac{1}{n}\right) q_{e}(y)<q_{e}(y) .
$$

Remark 2 The previous lemma can be viewed as a refinement of [14, Lemma 2.1].

In [16, Theorem 2], Huang and Zhang proved a cone metric version of celebrated Nemytzki-Edelstein fixed point theorem $([13,27])$ in the case when the underlying cone is regular. We will prove here that it holds true for arbitrary solid cones, with a modified contractive condition.

Theorem 1 Let $(X, p)$ be a tvs-cone metric space over a solid cone $C$ in $E$, and let $f: X \rightarrow$ $X$ be a mapping satisfying

$$
p(f x, f y) \ll p(x, y), \quad \text { for all } x \neq y .
$$

Suppose that there is $x_{0} \in X$ such that the respective Picard sequence $\left\{f^{n} x_{0}\right\}$ has a convergent subsequence (in particular, this is the case when $(X, p)$ is sequentially compact). Then $f$ has a unique fixed point, and for each positive integer $n$, it is $\operatorname{Fix}(f)=\operatorname{Fix}\left(f^{n}\right)$, i.e., $f$ has the property $P$.

Proof Take arbitrary $e \in \operatorname{int} C$ and form the respective monotone norm $q_{e}$ on $E$ according to Lemma 2. Denote $d=q_{e} \circ p$, i.e., $d(x, y)=q_{e}(p(x, y))$ for $x, y \in X$. Then $d$ is a metric on $X$ (by [20, Theorem 3.1]) and, using Lemma 3, the relation (1) implies that

$$
d(f x, f y)<d(x, y), \quad \text { for all } x \neq y .
$$


Now the proof proceeds similarly as in the metric case.

We will prove just the property $P$. Suppose that $u \in F i x\left(f^{n}\right)$, but $u \notin F i x(f)$, where $n$ is the smallest such index. Then

$$
d(u, f u)=d\left(f^{n} u, f^{n+1} u\right)<d\left(f^{n-1} u, f^{n} u\right)<\cdots<d(u, f u),
$$

a contradiction. Hence, $u \in F i x(f)$, i.e., $F i x\left(f^{n}\right) \subset$ Fix $(f)$. The reverse inclusion is obvious.

Formulated in another way:

Corollary 1 The scalar and vector (with « used in the condition (1)) versions of NemytzkiEdelstein fixed point theorem are equivalent.

Question 1 Do the conclusions of Theorem 1 hold if the cone C is just solid (and not regular) and $\ll$ in (1) is replaced by $\prec$ ?

\section{Tvs-cone $b$-metric spaces}

$b$-metric spaces (sometimes called metric-type spaces), as another generalization of metric spaces were first considered by I.A. Bakhtin [3] and Czerwik [8]. Cone $b$-metric spaces were introduced in [7] and [18]. Extension to tvs case can be done in an obvious way:

Definition 2 Let $X$ be a nonempty set, $(E, t, C)$ be an ordered tvs and $s \geq 1$ be a given real number. A function $p_{s}: X \times X \rightarrow E$ is called a tvs-cone $b$-metric and $\left(X, p_{s}\right)$ is called a tvs-cone $b$-metric space if the following conditions hold:

(ps1) $\theta \preceq p_{s}(x, y)$ for all $x, y \in X$ and $p_{s}(x, y)=\theta$ if and only if $x=y$;

(ps2) $p_{s}(x, y)=p_{s}(y, x)$ for all $x, y \in X$;

(ps3) $p_{s}(x, z) \preceq s\left[p_{s}(x, y)+p_{s}(y, z)\right]$ for all $x, y, z \in X$.

If $(E,\|\cdot\|, C)$ is an ordered normed space, then $p_{s}$ is called a cone b-metric, and $(X, d)$ is a cone b-metric space.

Obviously, for $s=1$, (tvs)-cone $b$-metric space is a (tvs)-cone metric space.

Most of the standard notions concerning convergence of sequences can be introduced in these spaces in the usual way. The main obstacle in deriving results is the fact that a (cone) $b$-metric is not always a continuous function (in the sense that $x_{n} \rightarrow x$ and $y_{n} \rightarrow y$ imply that $p_{s}\left(x_{n}, y_{n}\right) \rightarrow p_{s}(x, y)$ as $\left.n \rightarrow \infty\right)$, see, e.g., [17, Example 2]. 
Lemma 4 Let $\left(X, p_{s}\right)$ be a tvs-cone b-metric space (with the parameter $s$ ) over a solid cone $C, e \in \operatorname{int} C$ and let $q_{e}$ be the corresponding Minkowski functional of $[-e, e]$. Then $d_{q}=q_{e} \circ p_{s}$ is a b-metric on $X$ (with the same parameter $s$ ).

Proof Clearly, $d_{q}(x, y)=d_{q}(y, x)$ for all $x, y \in X$ and $x=y$ implies that $d_{q}(x, y)=0$. Also, since $q_{e}$ is a semi-norm and $p_{s}$ is a tvs-cone $b$-metric, we have

$$
q_{e}\left(p_{s}(x, z)\right) \leq s\left(q_{e}\left(p_{s}(x, y)\right)+q_{e}\left(p_{s}(y, z)\right)\right),
$$

i.e.,

$$
d_{q}(x, z) \leq s\left[d_{q}(x, y)+d_{q}(y, z)\right]
$$

for all $x, y, z \in X$. Now, we prove that $d_{q}(x, y)=0$ implies that $x=y$. Let $q_{e} \circ p_{s}(x, y)=0$. Then $\inf \left\{\lambda>0: p_{s}(x, y) \in \lambda[-e, e]\right\}=0$. Thus, there exists a sequence of positive scalars $\lambda_{n} \rightarrow 0$ such that $p_{s}(x, y) \in \lambda_{n}[-e, e]$. Suppose, to the contrary, that $x \neq y$. Then, since $\theta \prec p_{s}(x, y) \preceq \lambda_{n} e$, for each $c \in \operatorname{int} C$ there exists $n_{0}$ such that $p_{s}(x, y) \ll c$ for $n \geq n_{0}$. Since $c$ is an arbitrary interior point of the cone $C$ it follows that $p_{s}(x, y)=\theta$. This is a contradiction. Thus, the proof of lemma is complete.

Remark 3 Under the assumptions from the previous lemma, similarly as in [20, Theorem 3.2], the following properties can be easily deduced:

(i) A sequence $\left\{x_{n}\right\}$ converges to $x$ in $\left(X, p_{s}\right)$ if and only if $d_{q}\left(x_{n}, x\right) \rightarrow 0$ as $n \rightarrow \infty$;

(ii) $\left\{x_{n}\right\}$ is a Cauchy sequence in $\left(X, p_{s}\right)$ if and only if $\left\{x_{n}\right\}$ is a Cauchy sequence in $\left(X, d_{q}\right)$;

(iii) $\left(X, p_{s}\right)$ is complete if and only if $\left(X, d_{q}\right)$ is complete.

As a sample, we show how a tvs-cone $b$-metric version of Banach Contraction Principle can be easily deduced.

Theorem 2 Let $\left(X, p_{s}\right)$ be a complete tvs-cone b-metric space with $s \geq 1$ and $\lambda \in[0,1)$. If $f: X \rightarrow X$ satisfies the contractive condition

$$
p_{s}(f x, f y) \preceq \lambda p_{s}(x, y),
$$

for all $x, y \in X$, then $f$ has a unique fixed point in $X$. Moreover, for each $x \in X$, the Picard sequence $\left\{f^{n} x\right\}$ converges to the unique fixed point of $f$. 
Proof Set $d_{q}=q_{e} \circ p_{s}$. Lemma 4 and Remark 3 imply that $\left(X, d_{q}\right)$ is a complete $b$-metric space. Also, we conclude that (2) implies that

$$
d_{q}(f x, f y) \leq \lambda d_{q}(x, y)
$$

for all $x, y \in X$. Thus, the conclusion follows from [12, Theorem 2.1].

In a similar way, a lot of fixed point results under various contractive conditions can be proved in tvs-cone $b$-metric spaces, by reducing them to the respective results in $b$-metric spaces. Note that most of them need a modification compared with the respective standard metric results, in the sense that conditions on contractive constants depend on parameter $s$ (the case of Banach principle is an exception), see the respective discussion in [12].

We finish by applying our previous results in order to prove tvs-cone $b$-metric version of Nemytzki-Edelstein theorem in the case when tvs-cone $b$-metric is continuous. For discussion about (sequential) compactness in these spaces see [18].

Theorem 1 Let $\left(X, p_{s}\right)$ be a tvs-cone b-metric space over a solid cone $C$ in $E$, such that the tvs-cone $b$-metric $p_{s}$ is continuous. Let $f: X \rightarrow X$ be a mapping satisfying

$$
p_{s}(f x, f y) \ll p_{s}(x, y), \quad \text { for all } x \neq y .
$$

Suppose that there is $x_{0} \in X$ such that the respective Picard sequence $\left\{f^{n} x_{0}\right\}$ has a convergent subsequence (in particular, this is the case when $\left(X, p_{s}\right)$ is sequentially compact). Then $f$ has a unique fixed point.

Proof Similarly as in the proof of Theorem 1, take arbitrary $e \in \operatorname{int} C$ and form the respective monotone norm $q_{e}$ on $E$ according to Lemma 2. Denote $d_{q}=q_{e} \circ p_{s}$, i.e., $d_{q}(x, y)=$ $q_{e}\left(p_{s}(x, y)\right)$ for $x, y \in X$. Then, by Lemma $4, d_{q}$ is a $b$-metric on $X$ (with the same parameter $s$ as $\left.p_{s}\right)$. Moreover, $d_{q}$ is continuous, together with $p_{s}$. Applying Lemma 3, the relation (3) implies that

$$
d_{q}(f x, f y)<d_{q}(x, y), \quad \text { for all } x \neq y .
$$

Now the proof proceeds similarly as in the $b$-metric case (see, e.g., [6, Theorem 3.1]).

Question 2 Does the previous result hold true if the b-metric $p_{s}$ is not continuous?

\section{Acknowledgements}

The first and second authors are thankful to the Ministry of Education, Science and Technological Development of Serbia. 


\section{Competing interests}

The authors declare that they have no competing interests.

\section{Authors' contributions}

All authors contributed equally and significantly in writing this paper. All authors read and approved the final manuscript.

\section{References}

[1] C.D. Aliprantis, R. Tourky, Cones and Duality, Graduate Studies in Mathematics: American Mathematical Society, Providence, RI, 2007.

[2] A. Amini-Harandi, M. FaKhar, Fixed point theory in cone metric spaces obtained via scalarization method, Comput. Math. Appl. 59 (2010) 3529-3534.

[3] I.A. BAKhtin, The contraction mapping principle in quasi-metric spaces, Funct. Anal. Ulianowsk Gos. Ped. Inst. 30 (1989) 26-37.

[4] I. BEg, A. AZAM, M. ARSHAD, Common fixed points for maps on topological vector space valued cone metric spaces, Inter. J. Math. Math. Sci. 2009:560264.

[5] H. ÇAKAlLI, A. Sönmez, Ç. Genç, On an equivalence of topological vector space valued cone metric spaces and metric spaces, Appl. Math. Lett. 25 (2012) 429-433.

[6] M. Cosentino, P. SAlimi, P. Vetro, Fixed point results on metric-type spaces, Acta Math. Sci. 34B(4) (2014) 1237-1253.

[7] A.S. Cvetković, M.P. Stanić, S. Dimitrijević, Su. Simić, Common fixed point theorems for four mappings on cone metric type space, Fixed Point Theory Appl. 2011:589725.

[8] S. Czerwik, Contraction mappings in b-metric spaces, Acta Math. Inform. Univ. Ostrav. 1 (1993) 5-11.

[9] K. Deimling, Nonlinear Functional Analysis, Springer-Verlag, 1985.

[10] W.S. DU, A note on cone metric fixed point theory and its equivalence, Nonlinear Anal. 72 (5) (2010) 2259-2261.

[11] W. Du, E. KARAPINAR, A note on b-cone metric and its related results: Generalizations or equivalence, Fixed Point Theory Appl. 2013:210.

[12] N.V. Dung, V.T. LE HANG, On relaxations of contraction constants and Caristi's theorem in b-metric spaces, to appear in J. Fixed Point Theory Appl., DOI:10.1007/s11784-015-0273-9.

[13] M. Edelstein, An extension of Banach's contraction principle, Proc. Amer. Math. Soc. 12 (1961) 7-10.

[14] A.P. Farajzadeh, A. Amini-Harandi, D. Baleanu, Fixed point theory for generalized contractions in cone metric spaces, Commun. Nonlinear Sci. Numer. Simulat. 17 (2012) 708712. 
[15] Y. Feng, W. MaO, The equivalence of cone metric spaces and metric spaces, Fixed Point Theory 11 (2) (2010) 259-26.

[16] L.G. HuANG, X. ZHANG, Cone metric spaces and fixed point theorems of contractive mappings, J. Math. Anal. Appl. 332 (2007) 1467-1475.

[17] N. Hussain, V. Parvaneh, J.R. Roshan, Z. Kadelburg, Fixed points of cyclic $(\psi, \varphi, L, A, B)$-contractive mappings in ordered $b$-metric spaces with applications, Fixed Point Theory Appl. 2013:256.

[18] N. Hussain, M.H. Shah, KKM mappings in cone b-metric spaces, Comput. Math. Appl. 62 (2011) 1677-1684.

[19] S. JANKović, Z. KADELbURG, S. RAdEnOvić, On cone metric spaces: a survey, Nonlinear Anal. 74 (2011) 2591-2601.

[20] Z. KAdelbuRG, S. RADENOVIĆ, V. RAKoČEVIĆ, A note on the equivalence of some metric and cone metric fixed point results, Appl. Math. Lett. 24 (2011) 370-374.

[21] Z. Kadelburg, S. RAdenović, V. RaKočEvić, Topological vector space valued cone metric spaces and fixed point theorems, Fixed Point Theory Appl. 2010:170253.

[22] M.A. Khamsi, P.J. Wojciechows Ki, On the additivity of the Minkowski functionals, Nonlinear Funct. Anal. Optim. 34 (6) (2013) 635-647.

[23] M. Khani, M. Pourmahdian, On the metrizability of cone metric spaces, Topology Appl. 158 (2) (2011) 190-193.

[24] M. Krein, Propriétés fondamentales des ensembles coniques normaux dans l'espace de Banach, C.R. (Doklady) Acad. Sci. URSS. (N.S.) 28 (1940) 13-17.

[25] M.G. KReĬN, M.A. RUTMAN, Linear operators leaving invariant a cone in a Banach spaces [in Russian], Uspekhi Mat. Nauk. (N.S.) 3 (1) (1948) 3-95.

[26] Đ.R. KuREPA, Tableaux ramifiés d'ensembles, C.R. Acad. Sci. Paris. 198 (1934) 1563-1565.

[27] V.V. Nemytzki, The fixed point method in analysis [in Russian], Uspekhi Mat. Nauk. 1 (1936) 141-174.

[28] P.D. Proinov, A unified theory of cone metricspaces and its applications to the fixed point theory, Fixed Point Theory Appl. 2013:103.

[29] S. Rezapour, R. HAmlbarani, Some notes on the paper "Cone metric spaces and fixed point theorems of contractive mappings", J. Math. Anal. Appl. 345 (2008) 719-724.

[30] H.H. Schaefer, Topological Vector Spaces, 3rd ed., Berlin-Heidelberg-New York: SpringerVerlag, 1971.

[31] W. Yau-Chuen, NG. Kung-Fu, Partially Ordered Topological Vector Spaces, Clarendon Press, Oxford, 1973.

[32] P.P. Zabrejko, K-metric and K-normed linear spaces: survey, Collect. Math. 48 (1997), 825-859. 